Збірник наукових праць Українського державного університету залізничного транспорту

ЗАЛІЗНИЧНИЙ ТРАНСПОРТ (273)

УДК 629.4.015: 681.51

\begin{abstract}
МАТЕМАТИЧЕСКАЯ МОДЕЛЬ ПРОСТРАНСТВЕННЫХ КОЛЕБАНИЙ ЖЕЛЕЗНОДОРОЖНОГО ЧЕТЫРЕХОСНОГО АВТОНОМНОГО ТЯГОВОГО МОДУЛЯ
\end{abstract}

Ф. Буреші

\title{
MATHEMATICAL MODEL OF SPATIAL OSCILLATIONS OF A RAILWAY FOUR- AXLE AUTONOMOUS TRACTION MODULE
}

\section{František Bures}

\section{DOI: https://doi.org/10.18664/1994-7852.194.2020.230168}

Аннотация. В статье автором предложено описание оригинальной математической модели пространственных колебаний четырехосного автономного тягового модуля при его движении по прямолинейным и криволинейным участкам железнодорожного пути. При этом конструкиия четырехосного автономного тягового модуля представлена как сложная механическая система, а путь рассматривается как упруго-вязкая инериионная система. Разработанная автором математическая модель представляет собой систему дифференциальных уравнений 100-го порядка. В модели учитываются силь в связях между телами системы и пространственные перемещения твердых тел механической системы с учетом возможных ограничений. Данная математическая модель является базовой для проведения дальнейших теоретических исследований динамики железнодорожных четырехосных автономных тяговых модулей при одиночном движении или при движении в составе поезда.

Ключевые слова: автономный тяговый модуль,динамическая нагруженность, моделирование колебаний, динамические качества движения, транспортная механика.

Abstract. A description of the original mathematical model of spatial oscillations of a four-axle autonomous traction module during its movement along straight and curved sections of the railway track is proposed. In this case, the design of a four-axle autonomous traction module is presented as a complex mechanical system, and the track is considered as an elastic-viscous inertial system. The equations of motion were compiled using the Lagrange method of the II kind. For each of the solids, the kinetic energy is determined by the Koenig theorem. The potential energy component is obtained by the Clapeyron theorem, as the sum of the energies accumulated in the elastic elements of the system during their deformations. The dissipative energy was also taken into account when compiling the equations of motion. Generalized forces that have no potential, in this case, include the forces of interaction between wheels and rails, which are determined using the creep hypothesis.

It is important to note that the model takes into account the forces in the bonds between the bodies of the system and the spatial displacements of the rigid bodies of the mechanical system, taking into account possible restrictions.

Moreover, the mathematical model developed by the author is a system of differential equations of the 100th order. 
This mathematical model is the base for further theoretical studies of the dynamics of railway four-axle autonomous traction modules in single motion or when moving as part of a train.

To solve the resulting system of differential equations, the author develops special software that allows for complex theoretical studies of spatial oscillations of a four-axle autonomous traction module to determine the indicators of its dynamic loading and traffic safety.

Key words: autonomous traction module, dynamic loading, vibration simulation, dynamic motion qualities, transport mechanics.

Вступление. Обеспечение эффективности эксплуатации железнодорожного транспорта, как ведущей отрасли транспортной системы, требует внедрения современного подвижного состава. При этом особое внимание следует уделять рельсовым экипажам. При разработке новых конструкций рельсовых экипажей важное значение имеет оценка динамических качеств создаваемого транспортного средства и определение показателей безопасности движения. Особенно это актуально для тяговых рельсовых экипажей. В связи с этим важным является учет на стадии проектирования рельсовых экипажей уточненных динамических нагрузок, которые действуют на них в эксплуатации. Это вызывает необходимость создания уточненных математических моделей для определения динамических нагрузок, действующих на рельсовые экипажи в условиях эксплуатации.

Анализ последних исследований. Как правило, исследователями оценка динамических качеств рельсовых экипажей, как самоходных, так и несамоходных, производится с использованием математических моделей, которые учитывают основные конструктивные особенности рельсового экипажа $[1,2]$, его массовые и инерционные характеристики. Однако при этом важным является учет его движения не только в прямых участках пути, но и в кривых.

Для уточненной оценки динамических качеств рельсовых экипажей в работах $[3,4]$ учитываются типы связей между элементами конструкции. При этом чаще всего используются упругие, упругофрикционные или вязкие связи.
Необходимо отметить, что во внимание следует принимать перемещения рельсовых экипажей не только в плоской системе координат, а и в пространственной.

В работах [5, 6] освещаются особенности создания математических моделей перемещений рельсовых экипажей. При этом движение рельсовых экипажей рассматривается в вертикальной и продольной плоскостях. Составление математических моделей их движения осуществляется с использованием метода Лагранжа II рода. Важно отметить, что для более точной оценки динамических качеств рельсовых экипажей необходимым является использование более современных методов и средств решений математических моделей их перемещений.

При математическом моделировании перемещений тяговых рельсовых экипажей необходим также учитывать конструктивное расположение и характер опирания тяговых двигателей на механическую часть, то есть на элементы тележек, что также требует определенного математического описания [6-8]. Однако при решении таких математических моделей предпочтительней использование средств компьютерного моделирования, что

позволит получить более точную оценку динамических качеств рельсовых экипажей.

Для формирования математических моделей пространственных колебаний рельсовых экипажей и исследования взаимодействия подвижного состава и пути в работе [9] используются различные теории по определению сил проскальзывания между колесом и рельсом. При этом в работе не учитывается перемещение рельсовых экипажей в кривых. 
Различные аспекты формирования математических моделей тяговых рельсовых экипажей с учетом взаимодействия механических частей и контакта не только с рельсовой колеей, но и с контактным проводом для электроподвижного состава железных дорог, а также с учетом свойств ударнотяговых устройств и поглощающих аппаратов представлены в работах [10 - 12]. Однако при этом математические модели учитывают перемещение рельсовых экипажей в прямых участках пути.

Определение цели и задачи исследования. Целью данного исследования является создание математической модели для изучения динамической нагруженности четырехосного автономного тягового модуля при движении в одиночном режиме и в составе поезда.

Для достижения поставленной цели определены такие задачи:

1. Выбор базовой расчетной схемы;

2. Математическое описание возможных колебаний тел системы;

3. Определение ограничений и видов связей между телами системы, включая взаимодействие экипажа и рельсового пути;

4. Составление системы дифференциальных уравнений пространственных колебаний автономного тягового модуля в четырехосном исполнении.

Основная часть исследования. Далее рассмотрим порядок формирования математической модели пространственных колебаний железнодорожного четырехосного автономного тягового модуля. Данный вариант конфигурации автономного транспортного средства выбран исходя из предварительных исследований, проведенных автором, по определению характеристик конструкции тягового средства для использования при транспортировке железнодорожных поездов по направлению «Восток - Запад», a точнее по направлению «Шелкового пути». При этом учитывались как механические особенности конструкции, так и тягово-энергетические параметры автономных тяговых модулей. В данном исследовании рассмотрим движение автономного тягового модуля как механической системы, то есть совокупности твердых тел, связанных между собой системами гашения колебаний и элементами крепления или соединениями различного типа. В начале выполним описание выбранной расчетной схемы.

Расчетную схему четырехосного автономного тягового модуля как механическую конструкцию можно представить в виде системы следующих основных элементов: кузов, тележки и колесные пары. Кузов автономного тягового модуля установлен на двух двухосных тележках. Соединения кузова с рамой тележки обеспечивают возможность поворота тележек относительно кузова в горизонтальной плоскости, что создает момент трения, необходимый для гашения колебаний виляния тележек (пятниковое соединение). Для ограничения колебаний кузова относительно тележек в вертикальной плоскости на рамах тележек установлены скользуны по аналогии с основными типами локомотивов [1-3].

Основными элементами двухосной тележки являются колесные пары и рама. Двухосная тележка оснащена индивидуальным тяговым приводом на каждую ось с опорно-осевым подвешиванием двигателя. Конструкция тележки имеет буксовое (первая ступень) и центральное (вторая ступень) рессорные подвешивания с использованием упругих элементов и гасителей колебаний. Буксовое рессорное подвешивание обеспечивает опору рамы тележки на каждую буксу через два пакета цилиндрических пружин. Для демпфирования вертикальных колебаний параллельно пружинам между буксами и рамой тележки установлено по одному гидравлическому гасителю колебаний на буксовый узел. Кузов опирается на рамы тележки через комплекты двухрядных цилиндрических пружин. Отклонения 
тележек относительно кузова в поперечном направлении ограничиваются упругими боковыми упорами, т. е. характеристика поперечной связи кузова с тележками является существенно нелинейной.

В данной математической модели рассматривается движение автономного тягового модуля по упруго-вязкому инерционному пути, который моделируется приведенной к каждому колесу массой (восемь приведенных масс), имеющей только вертикальное и поперечное горизонтальное перемещения и опирающейся в этих направлениях на пружины и демпферы вязкого трения, моделирующие упругодиссипативные свойства рельсов и подрельсового основания.

Расчетная схема автономного тягового модуля построена как механическая система, которая состоит из девятнадцати твердых тел: кузов, две рамы, четыре двигателя, четыре колесных пары и восемь приведенных масс пути, имеющих только вертикальные и поперечные горизонтальные перемещения. Таким образом, в общем случае система имеет $11 \times 6+8 \times 2=82$ степени свободы.

При описании движения рассматриваемого экипажа по участкам пути произвольного очертания выберем неподвижную систему координат $\tilde{O} \xi \eta \zeta$, a для каждого твердого тела - по две подвижные системы координат: естественную $O x y z$ и связанную с твердым телом $C x^{\prime} y^{\prime} z^{\prime}\left(C x^{\prime}, C y^{\prime}, C z^{\prime}\right.$ - главные центральные оси инерции). Все системы координат примем правыми, причем будем считать, что оси $\widetilde{O} \xi, O x, C x^{\prime}$ направлены слева направо, а оси $\widetilde{O} \zeta, O z, C z^{\prime}-$ вниз, как представлено на рисунке.

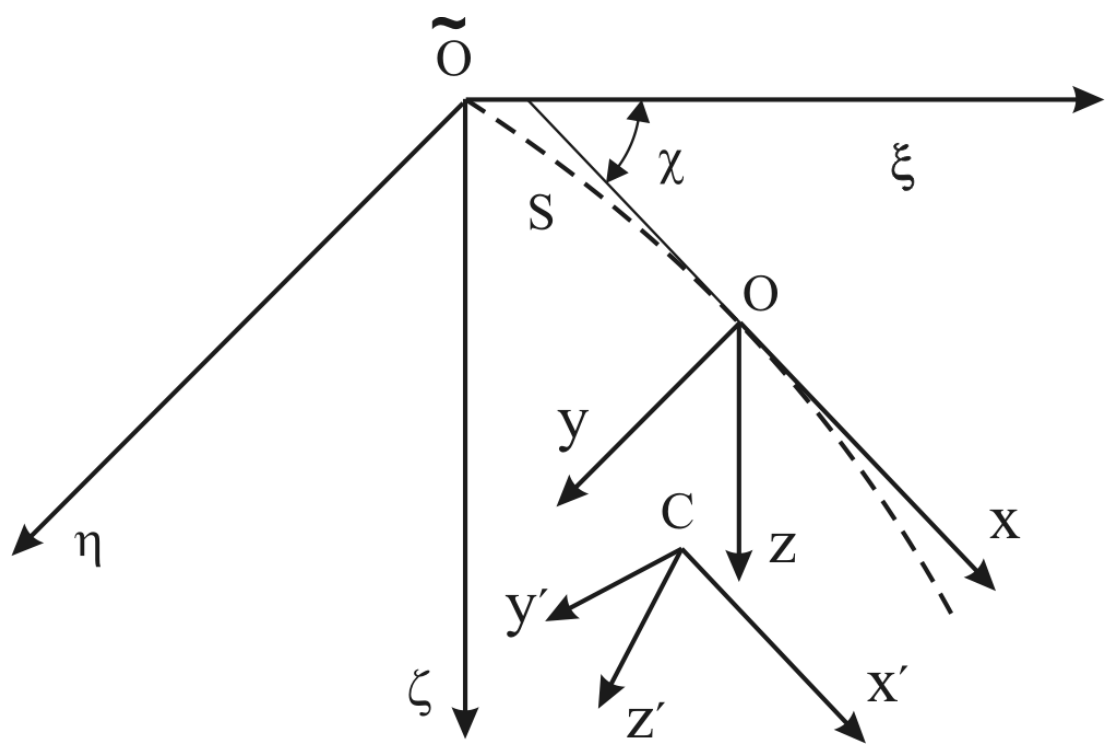

Рис. Системы координат расчетной схемы автономного тягового модуля: $\widetilde{O} \xi \eta \zeta$ - неподвижная система координат; Oxyz - подвижная естественная система координат; $C x^{\prime} y^{\prime} z^{\prime}$ - подвижная система координат, связанная с твердым телом; $\mathrm{S}$ - траектория движения автономного тягового модуля; $\chi$ - угол между осями $\widetilde{O} \zeta$ и $O x$

Оси естественной системы координат направлены соответственно по касательной, нормали и бинормали к оси пути. Начало координат $O$ для каждого твердого тела находится на расстоянии $S$ от его 
положения в начальный момент времени (здесь $S$ - пройденный путь). Положение естественной системы координат относительно неподвижной характеризуется дуговой координатой вдоль оси пути $s$, углом $\chi$ между осями $O x$ и $\widetilde{O} \xi$ в плане, а также углами $\varphi_{h}$ и $\theta_{h}$ между этими осями в вертикальных плоскостях, которые определяются возвышением наружного рельса $h_{r}$. Параметры рельса $\chi, h_{r}$ заданные функции координаты $s$, для круговой кривой $\chi \chi \chi$ и $h_{r}$ - постоянные, для прямой - приравниваются нулю [4, 13].

При выводе уравнений движения использованы общепринятые обозначения перемещений отдельных тел системы $x, y, z, \psi, \varphi, \theta[5,14,15]$. Поступательные перемещения $x, y, z$ и углы поворота $\psi, \varphi, \theta$ описывают соответственно подергивание, боковой относ, подпрыгивание и виляние, галопирование, боковую качку твердого тела.

При обозначении координат твёрдых тел системы введены следующие индексы: кузов - $f$, рама тележки $-s l(l=1,2)$, двигатели $-b(b=\overline{1,4})$, колёсная пара $-i$ $(i=\overline{1,4}$ - номер колёсной пары по ходу движения экипажа), колесо - $i j$ ( $j=1$ левая сторона вагона, $j=2-$ правая), рельсы в точках контакта - rij .

При составлении уравнений движения автономного тягового модуля приняты во внимание следующие конструктивные особенности:

- действие сил сухого трения, возникающих при взаимных перемещениях кузова и тележки в плане;

- упруго-вязкая связь кузова с рамой тележки;

- нелинейная характеристика силы в пятниковом узле;

- упруго-вязкая связь рамы тележки с колесными парами;

- наличие дополнительной связи между рамой тележки и осью колесной пары, вызванное наличием двигателей, имеющих опорно-рамное подвешивание;

- криволинейность профилей поверхностей катания колеса и рельса, а также нелинейность сил взаимодействия между ними.

Для определения числа степеней свободы рассматриваемой механической системы приняты во внимание ограничения, наложенные на перемещения тел, с учетом общепринятых допущений и конструктивных особенностей ходовых частей автономного тягового модуля, по аналогии с тяговым подвижным составом [6].

Вертикальные перемещения и боковая качка колесных пар выражаются через вертикальные перемещения колес

$$
z_{i}=\frac{{ }_{i 1}+z_{i 2}}{2} ; \quad \theta_{i}=\frac{{ }_{i 2}-z_{i 1}}{2 d_{1}} ;(i=\overline{1,4}),
$$

где $2 d_{1}-$ расстояние между средними кругами катания колесной пары.

Предполагается, что радиусы $r$ кругов катания всех колес равны между собой. Тогда при совпадении продольных плоскостей симметрии пути и тележки при перемещении автономного тягового модуля вдоль оси пути все колеса повернутся на один и тот же угол

$$
\varphi_{i}=-\frac{x_{i}+s}{r},(i=1,4) .
$$

Двигатели во всех направлениях, кроме галопирования, движутся совместно с рамой тележки:

$$
\begin{aligned}
& x_{s l}=x_{b} ; \\
& y_{s l}=y_{b} ; \\
& z_{s l}=z_{b} ; \\
& \theta_{s l}=\theta_{b} ; \\
& \psi_{s l}=\psi_{b} \quad(b=\overline{1,4}) .
\end{aligned}
$$


С учетом полученных связей (1)-(3) система «автономный тяговый модуль рельсовая колея» имеет $82-32=50$ степеней свободы.
Обобщенные координаты, описывающие конфигурацию рассматриваемой механической системы, выбраны следующим образом:

$$
\begin{array}{ccccc}
q_{1}=y_{f} ; & q_{2}=y_{s 1} ; & q_{3}=y_{s 2} ; & q_{4}=y_{1} ; & q_{5}=y_{2} ; \\
q_{6}=y_{3} ; & q_{7}=y_{4} ; & q_{8}=\psi_{f} ; & q_{9}=\psi_{s 1} ; & q_{10}=\psi_{s 2} ; \\
q_{11}=\psi_{1} ; & q_{12}=\psi_{2} ; & q_{13}=\psi_{3} ; & q_{14}=\psi_{4} ; & q_{15}=\theta_{f} ; \\
q_{16}=\theta_{s 1} ; & q_{17}=\theta_{s 2} ; & q_{18}=z_{f} ; & q_{19}=z_{s 1} ; & q_{20}=z_{s 2} ; \\
q_{21}=\varphi_{f} ; & q_{22}=\varphi_{s 1} ; & q_{23}=\varphi_{s 2} ; & q_{24}=x_{f} ; & q_{25}=x_{s 1} ; \\
q_{26}=x_{s 2} ; & q_{27}=x_{1} ; & q_{28}=x_{2} ; & q_{29}=x_{3} ; & q_{30}=x_{4} ; \\
q_{31}=\varphi_{b 1} ; & q_{32}=\varphi_{b 2} ; & q_{33}=\varphi_{b 3} ; & q_{34}=\varphi_{b 4} ; \\
q_{35}=y_{r 11} ; & q_{36}=y_{r 12} ; & q_{37}=y_{r 21} ; & q_{38}=y_{r 22} ; & q_{39}=y_{r 31} ; \\
q_{40}=y_{r 32} ; & q_{41}=y_{r 41} ; & q_{42}=y_{r 42} ; & q_{43}=z_{r 11} ; & q_{44}=z_{r 12} ; \\
q_{45}=z_{r 21} ; & q_{46}=z_{r 22} ; & q_{47}=z_{r 31} ; & q_{48}=z_{r 32} ; & q_{49}=z_{r 41} ; \\
q_{50}=z_{r 42} ; & & &
\end{array}
$$

Дифференциальные уравнения движения четырехосного автономного тягового модуля как сложной механической системы составлены в форме уравнений Лагранжа второго рода $[7,16,17]$

$$
\frac{d}{d t}\left(\frac{\partial T}{\partial q_{i}}\right)-\frac{\partial T}{\partial q_{i}}+\frac{\partial \Pi}{\partial q_{i}}+\frac{\partial \Phi}{\partial q_{i}}=Q_{i}+S_{i}^{*}, \quad(i=1,50)
$$

где $T$ и $\Pi$ - кинетическая и потенциальная энергии системы;

$\Phi$ - функция рассеяния;

$q_{i}, q_{i}$ - обобщенные координаты и их скорости;

$Q_{i}$ - соответствующие им обобщенные силы, не имеющие потенциала;

$S_{i}^{*}$ - приложенные внешние силы.
Кинетическую энергию автономного тягового модуля $(T)$ определим как сумму энергий всех твердых тел, входящих в систему.

Для каждого из твердых тел кинетическая энергия определяется по теореме Кенига. В общем случае выражение кинетической энергии для $i$-го твёрдого тела записывается в следующем виде:

$$
\begin{aligned}
& \left.T={ }_{i} m_{i}\left[\left(s+\bar{x}-y_{i} \bar{\chi}_{i}\right)^{2}+\left(y_{i}+x \bar{\chi}_{i}\right)_{i}^{2}+\left(z_{i}-h\right)_{r i}\right)^{2}\right]+ \\
& \frac{1}{2} I_{x i}\left(\theta_{i}+\bar{\theta}_{h i}\right)^{2}+\frac{1}{2} I_{z i}\left(\psi_{i}+\bar{\chi}_{i}\right)^{2}+\frac{1}{2}_{y i}\left(\varphi_{i}+\varphi_{h i}\right)^{2},
\end{aligned}
$$

где $m_{i}$ - масса $i$-го твёрдого тела;

$I_{i}$ с соответствующими индексами обозначают главные центральные моменты инерции $i$-го тела; 
$\bar{\chi}_{i}=v K_{i}, K_{i}-$ кривизна пути под $i$-м телом;

$v$ - скорость движения;

$S$ - пройденный путь;

$h_{r i}$ - возвышение пути под центром масс $i$-го тела, вызванное возвышением в кривой наружного рельса на величину $h_{r}=\theta_{h} 2 d_{1}$.

Кинетическая энергия системы, моделирующей путь, в соответствии с принятыми допущениями записывается следующим образом:

$$
T_{t}=\frac{1}{2} m_{r h} \sum_{i=1}^{4} \sum_{j=1}^{2} y_{r i j}^{2}+\frac{1}{2} m_{r v} \sum_{i=1}^{4} \sum_{j=1}^{2} z_{r i j}^{2},
$$

где $m_{r h}, m_{r v}-$ массы пути, приведенные к одному колесу в горизонтальном поперечном и вертикальном направлениях.

Потенциальная энергия рассматриваемой системы (П) равна сумме энергии сжатия упругих элементов подвешивания $\left(\Pi_{1}\right)$ и потенциала сил тяжести $\left(\Pi_{2}\right)$

$$
\Pi=\Pi_{1}+\Pi_{2} .
$$

Составляющая потенциальной энергии $\Pi_{1}$ определяется по теореме Клапейрона как сумма энергий, накапливаемых в упругих элементах системы при их деформациях, следующим образом:

$$
\Pi_{1}=\frac{1}{z}\left[\begin{array}{l}
{\left[\sum_{i=1}^{4}\left(k_{c z i} \Delta_{c z i}^{2}+k_{c y i} \Delta_{c y i}^{2}+k_{c x i} \Delta_{c x i}^{2}\right)+\sum_{i=1}^{8}\left(k_{a z i} \Delta_{a z i}^{2}+k_{a y i} \Delta_{a y i}^{2}+k_{a x i} \Delta_{a x i}^{2}\right)+\right.} \\
{\left[+k_{s} \sum_{i=1}^{4} \Delta_{b i}+k_{z} \sum_{i=1}^{4} \Delta_{z i}+\sum_{i=1}^{4} \sum_{j=1}^{2}\left(k_{r z} \Delta_{r z i j}^{2}+k_{r y} \Delta^{2} r y i j\right)\right.}
\end{array}\right\rfloor,
$$

где $k_{c z i}, k_{c y i}, k_{c x i} \quad$ - соответственно жесткости $i$-го упругого элемента центрального подвешивания тележки в вертикальном, горизонтальном поперечном и продольном направлениях;

$$
\Delta_{c z i}, \Delta_{c y i}, \Delta_{c x i} \quad-\quad \text { соответственно }
$$
вертикальный, горизонтальный поперечный и продольный прогибы $i$-го упругого элемента центрального подвешивания;

$$
k_{a z i}, k_{a y i}, k_{a x i}-\text { жесткости } i \text {-го упругого }
$$
элемента буксового подвешивания тележки в вертикальном, горизонтальном поперечном и продольном направлениях;

$\Delta_{a z i}, \Delta_{a y i}, \Delta_{a x i}$ - вертикальный, горизонтальный поперечный и продольный прогибы $i$-го упругого элемента буксового подвешивания;

$k_{s}$ - угловая жесткость соединения двигателя с рамой тележки;

$\Delta_{b i}$ - угловая деформация соединения $i$ го двигателя с рамой тележки; $k_{z}$ - жесткость скользуна, установленного на раме тележки, ограничивающего вертикальные перемещения кузова;

$\Delta_{z i}$ - взаимное вертикальное перемещение кузова и рамы тележки в зоне $i$-го скользуна;

$k_{r z}, k_{r y}-$ вертикальная и горизонтальная поперечная жесткости рельсовой колеи;

$\Delta_{r z i j}, \Delta_{r y i j}-$ вертикальный и горизонтальный поперечный прогибы рельсовой колеи под $j$-м колесом $i$-й колесной пары.

Потенциальная энергия, обусловленная опусканием центром тяжести $i$-го твёрдого тела, с учетом криволинейного движения определяется следующим образом:

$$
\Pi_{2}=-\sum_{i=1}^{7} m_{i} g\left(\theta_{h i} y_{i}+z_{i}\right),
$$

где $g$ - ускорение свободного падения; 


\section{$\theta_{h i} \quad-$ угол между горизонтальной} плоскостью под центром масс $i$-го тела и плоскостью пути, вызванный возвышением наружного рельса над внутренним.

\section{Взаимные перемещения тел, вызывающих деформации упругих элементов, определяются следующим образом: \\ - вертикальные прогибы упругих элементов центрального подвешивания:}

$$
\begin{aligned}
& \Delta_{c z 1}=z_{f}-l_{1} \varphi_{f}-z_{s 1}+b\left(\theta_{f}-\theta_{s 1}+\theta_{h f}-\theta_{h s 1}\right) ; \\
& \Delta_{c z 2}=z_{f}-l_{1} \varphi_{f}-z_{s 1}-b\left(\theta_{f}-\theta_{s 1}+\theta_{h f}-\theta_{h s 1}\right) ; \\
& \Delta_{c z 3}=z_{f}+l_{2} \varphi_{f}-z_{s 2}+b\left(\theta_{f}-\theta_{s 2}+\theta_{h f}-\theta_{h s 2}\right) ; \\
& \Delta_{c z 4}=z_{f}+l_{2} \varphi_{f}-z_{s 2}-b\left(\theta_{f}-\theta_{s 2}+\theta_{h f}-\theta_{h s 2}\right),
\end{aligned}
$$

где $l_{1}, l_{2}$ - расстояние от центра масс кузова до центра масс первой и второй тележки в продольном направлении соответственно;

\section{$2 b$ - расстояние между упругими} элементами центрального подвешивания в поперечном направлении;

- горизонтальные п поперечные прогибы упругих элементов центрального подвешивания:

$$
\begin{aligned}
& \Delta_{c y 1}=\Delta_{c y 2}=y_{f}+l_{1} \psi_{f}-h \theta_{f}-y_{s 1}-u ; \\
& \Delta_{c y 3}=\Delta_{c y 4}=y_{f}-l_{2} \psi_{f}-h \theta_{f}-y_{s 2}-u,
\end{aligned}
$$

где $h$ - расстояние от центра масс кузова автономного тягового модуля до плоскости опирания рам тележек на упругие элементы центрального подвешивания;

$$
u=\frac{1}{2}\left(l_{1}+l_{2}\right)^{2} K_{f}-\text { стрела дуги криволи- }
$$

нейного (в плане) пути под центром масс кузова в пределах геометрической базы автономного тягового модуля $\left(l_{1}+l_{2}\right)\left(K_{f}\right.$

- кривизна пути под центром масс кузова автономного тягового модуля);

$$
\text { - горизонтальные }
$$

продольные прогибы упругих элементов центрального подвешивания:

$$
\begin{aligned}
& \Delta_{c x i}=x_{f}-x_{s 1}+(-1)^{i} b\left(\psi_{f}-\psi_{s 1}+\chi_{f}-\chi_{s 1}\right)-(-1)^{i} l K_{f} \quad(i=\overline{1,2}) ; \\
& \Delta_{c x i}=x_{f}-x_{s 2}+(-1)^{i} b\left(\psi_{f}-\psi_{s 2}+\chi_{f}-\chi_{s 2}\right)-(-1)^{i} l K_{f} \quad(i=\overline{3,4}),
\end{aligned}
$$

где $\chi_{j}-$ текущий угол между продольной осью неподвижной системы координат для $j$-го твердого тела и осью $O x$ естественной системы координат;

- вертикальные прогибы первой ступени подвешивания:

$$
\begin{aligned}
& \Delta_{a z i}=z_{s 1}-a \varphi_{s 1}-z_{1}-(-1)^{i} b^{*}\left(\theta_{s 1}-\theta_{1}+\theta_{h s 1}-\theta_{h 1}\right),(i=1,2) ; \\
& \Delta_{a z i}=z_{s 1}+a \varphi_{s 1}-z_{2}-(-1)^{i} b^{*}\left(\theta_{s 1}-\theta_{2}+\theta_{h s 1}-\theta_{h 2}\right),(i=3,4) ; \\
& \Delta_{a z i}=z_{s 2}-a \varphi_{s 2}-z_{3}-(-1)^{i} b^{*}\left(\theta_{s 2}-\theta_{3}+\theta_{h s 2}-\theta_{h 3}\right),(i=5,6) ;
\end{aligned}
$$




$$
\Delta_{a z i}=z_{s 2}+a \varphi_{s 2}-z_{4}-(-1)^{i} b^{*}\left(\theta_{s 2}-\theta_{4}+\theta_{h s 2}-\theta_{h 4}\right),(i=7,8),
$$

где $2 a$ - база тележки;

$2 b^{*}$ - расстояние между упругими элементами буксового подвешивания в поперечном направлении;

- горизонтальные поперечные прогибы первой ступени подвешивания:

$$
\begin{aligned}
& \Delta_{a y 1}=\Delta_{a y 2}=y_{s 1}+a \psi_{s 1}-y_{1}-u_{1}^{*} ; \\
& \Delta_{a y 3}=\Delta_{a y 4}=y_{s 1}-a \psi_{s 1}-y_{2}-u_{1}^{*} ; \\
& \Delta_{a y 5}=\Delta_{a y 6}=y_{s 2}+a \psi_{s 2}-y_{3}-u_{2}^{*} ; \\
& \Delta_{a y 7}=\Delta_{a y 8}=y_{s 2}-a \psi_{s 2}-y_{4}-u_{2}^{*},
\end{aligned}
$$

где $u_{1}^{*}, u_{2}^{*}-$ стрелы дуг криволинейного (в плане) пути в пределах баз первой и второй тележек;

- продольные прогибы первой ступени подвешивания:

$$
\begin{aligned}
& \Delta_{a x i}=x_{s 1}-x_{1}+(-1)^{i} b^{*}\left(\psi_{s 1}-\psi_{1}+\chi_{s 1}-\chi_{1}\right)-(-1)^{i} a K_{s 1},(i=1,2) ; \\
& \Delta_{a x i}=x_{s 1}-x_{2}+(-1)^{i} b^{*}\left(\psi_{s 1}-\psi_{2}+\chi_{s 1}-\chi_{2}\right)-(-1)^{i} a K_{s 1}(i=3,4) ; \\
& \Delta_{a x i}=x_{s 2}-x_{3}+(-1)^{i} b^{*}\left(\psi_{s 2}-\psi_{3}+\chi_{s 2}-\chi_{3}\right)-(-1)^{i} a K_{s 2}(i=5,6) ; \\
& \Delta_{a x i}=x_{s 2}-x_{4}+(-1)^{i} b^{*}\left(\psi_{s 2}-\psi_{4}+\chi_{s 2}-\chi_{4}\right)-(-1)^{i} a K_{s 2},(i=7,8) ;
\end{aligned}
$$

- деформации соединения двигателя с рамой тележки:

$$
\begin{aligned}
& \Delta_{1}=\varphi_{s 1}-\varphi_{b 1} ; \\
& \Delta_{2}=\varphi_{s 1}-\varphi_{b 2} ; \\
& \Delta_{3}=\varphi_{s 2}-\varphi_{b 3} ; \\
& \Delta_{4}=\varphi_{s 2}-\varphi_{b 4} ;
\end{aligned}
$$

- взаимные вертикальные перемещения кузова и рам тележек в зоне расположения скользунов:

$$
\begin{aligned}
& \Delta_{z 1}=z_{f}-z_{s 1}-b_{s}\left(\theta_{f}+\theta_{h f}-\theta_{s 1}-\theta_{h s 1}\right) ; \\
& \Delta_{z 2}=z_{f}-z_{s 1}+b_{s}\left(\theta_{f}+\theta_{h f}-\theta_{s 1}-\theta_{h s 1}\right) ; \\
& \Delta_{z 3}=z_{f}-z_{s 2}-b_{s}\left(\theta_{f}+\theta_{h f}-\theta_{s 2}-\theta_{h s 2}\right) ; \\
& \Delta_{z 4}=z_{f}-z_{s 2}+b_{s}\left(\theta_{f}+\theta_{h f}-\theta_{s 2}-\theta_{h s 2}\right),
\end{aligned}
$$


где $2 b_{s}$ - расстояние между скользунами в поперечном направлении;

- прогибы пути:

$$
\begin{aligned}
& \Delta_{r z i j}=z_{r i j}-z_{i j} ; \\
& \Delta_{r y i j}=y_{r i j}, \quad(i=\overline{1,4} ; j=1,2) .
\end{aligned}
$$

В построенной расчетной схеме введены гидравлические гасители колебаний, установленные в обеих ступенях подвешивания. Кроме того, учитывается действие сил сухого трения при взаимных поворотах кузова автономного тягового модуля и рам тележек в плане. При

$$
\begin{aligned}
& \Phi={ }^{1^{4}{ }^{2}}\left(\beta \Delta^{2}+\beta \Delta^{2}\right)+{ }^{1{ }^{4}}\left(\beta \Delta^{2}+\beta \Delta^{2}\right)+ \\
& \overline{2} \sum_{i=1} \sum_{j=1} \quad r z \quad r z i j \quad r y \quad r y i j \quad \overline{2} \sum_{i=1} \quad c z i \quad c z i \quad c y i \quad c y i \quad \text {, } \\
& +\sum_{i=1}^{8}\left(\beta_{a z i}{ }_{a z i}^{2}+\beta_{a y i}{ }_{a y i}^{2}\right)+\sum_{i=1}^{2} W_{i}\left|\bar{A}_{\psi i}\right|,
\end{aligned}
$$

где $\beta_{r z}, \beta_{r y}-$ коэффициенты рассеяния энергии в пути в вертикальном и горизонтальном поперечном направлениях;

$\beta_{c y i}, \beta_{c z i}-$ коэффициенты вязкого трения $i$-го гидравлического гасителя колебаний, установленного в центральной ступени подвешивания в вертикальном и горизонтальном поперечном направлениях соответственно;

$$
\beta_{a z i}, \beta_{a y i}-\text { коэффициенты вязкого }
$$

трения $i$-го гидравлического гасителя колебаний, установленного в буксовой ступени подвешивания в вертикальном и горизонтальном поперечном направлениях соответственно;

$W_{i}$ - момент сил сухого трения, действующих в горизонтальной плоскости при взаимных поворотах кузова автономного тягового модуля и рамы $i$-й тележки в пятниковой зоне;

$\Delta$ - относительные скорости перемещений тел, соединенных гидравлическими гасителями колебаний;

$\bar{\Delta}_{\psi i}$ - скорость взаимных угловых перемещений в плане кузова автономного тягового модуля и рамы $i$-й тележки в шкворневом узле $\left(\bar{\Delta}_{\psi i}=\bar{\psi}_{f}+\bar{\chi}_{f}-\bar{\psi}_{s i}-\bar{\chi}_{s i}\right)$.

Относительные скорости $\Delta$ перемещений тел, соединенных диссипативными элементами, определяются как производные по времени от относительных перемещений соответствующих тел.

Обобщенные силы $Q_{i}$, не имеющие потенциала, включают в себя силы взаимодействия колес и рельсов, которые определяются с использованием гипотезы крипа. Согласно этой гипотезе касательные силы взаимодействия колес с рельсами зависят нелинейно от безразмерных характеристик проскальзывания. Характеристики и величина сил, действующих в контакте колеса и рельса, определяются по методике, описанной в работе [8].

Составляющие сил крипа определяются по формулам 


$$
\begin{gathered}
T_{x i j}=\frac{\varepsilon_{x i j}}{\varepsilon_{i j}} T_{i j} ; \\
\varepsilon=\frac{\varepsilon_{y i j} \sec \alpha_{i j}}{\varepsilon} T \underbrace{}_{i j} \quad(i=\overline{1,4},
\end{gathered}
$$

где $T_{x i j}$ - проекция полной силы $T_{i j}$ на продольную ось $x$;

$T_{\text {кij }} \quad-$ проекция силы $T_{i j}$ на касательную к профилю поверхности колеса в точке контакта;

$$
\alpha_{i j}-\text { угол между касательной к }
$$

профилю поверхности колеса в точке контакта и поперечной осью $y$;

$\varepsilon_{i j}-$ относительная скорость проскальзывания $j$-го колеса $i$-й колесной пары по рельсу (крип);

$$
\varepsilon_{x i j}, \varepsilon_{y i j}-\text { продольная и поперечная }
$$

составляющие относительной скорости

проскальзывания $j$-го колеса $i$-й колесной пары по рельсу;

$$
\begin{aligned}
& \left\lceil F_{i j} \varepsilon_{i j} 4\right\rceil-\underline{4} \\
& T_{i j}=-\left.F_{i j} \varepsilon_{i j}\right|_{\|}\left(f_{t r} N_{i j}\right)+1 \downarrow \text {, }
\end{aligned}
$$

где $N_{i j}$ - нормальное давление $j$-го колеса $i$-й колесной пары на рельс;

$f_{t r}$ - коэффициент трения скольжения;
$F_{i j}$ - коэффициент крипа $j$-го колеса $i$-й колесной пары.

Полный крип $\varepsilon_{i j} \quad$ определяется выражением [9]

$$
\varepsilon_{i j}=\left(\varepsilon_{x i j}^{2}+\varepsilon_{y i j}^{2} \sec ^{2} \alpha_{i j}\right)^{\frac{1}{2}} .
$$

Для рассматриваемой системы безразмерные характеристики проскальзываний, необходимые для определения сил псевдоскольжения, определяются следующим образом [7]

$$
\begin{aligned}
\varepsilon_{x i j} & =\left(\bar{x}_{i}+(-1)^{j} d \psi_{1} \psi_{i}\right) V_{i}^{-1}-\Delta r \underset{i j}{r^{-1}}+(-1)^{j+1} d K_{i} K_{i}(i=1,4 ; j=1,2) . \\
\varepsilon_{y i j} & ={ }_{\bar{s}}^{1}\left(y_{i}-r \theta_{i j}-y_{r i j}\right)-\psi_{i} \quad(i=1,4, j=1,2),
\end{aligned}
$$

где $d_{i j}^{*}-$ расстояние в поперечном направлении между текущей точкой контакта на $j$-м колесе $i$-й колесной пары и плоскостью симметрии колесной пары;

$r_{i j}$ - текущий радиус колеса, соответствующий положению точки контакта, который равен $r_{i j}=r+\Delta r_{i j}$;

$\Delta r_{i j}-$ приращение радиуса круга катания $j$-го колеса $i$-й колесной пары в рассматриваемой точке контакта.

Обобщенные силы $Q_{i}$ вычисляются как коэффициенты при вариациях обобщенных координат в выражениях возможных работ сил псевдоскольжения $T_{x i j}, T_{\alpha i j}$.
Обобщенные силы $Q_{i}$ получены после подстановки (5 - 7) в (4) и составления возможных работ сил взаимодействия колес и рельсов.

Внешними силами $S^{*}$, действующими на автономный тяговый модуль, являются сила тяги $F_{T}$, развиваемая автономным тяговым модулем, тормозная сила $B_{T}$, возникающая при включении тормозов, и силы сопротивления движению $W$, к которым относят все остальные внешние силы.

В общем случае внешние силы, действующие на автономный тяговый модуль, могут быть представлены в следующем виде:

$$
S_{i}^{*}=W+B+F
$$


где $W_{i}$ - сила сопротивления поступательному движению, приложенная к $i$-му твердому телу;

$B_{i}$ - тормозная сила, приложенная к $i$-му твердому телу;

$F_{T}$ - сила тяги автономного тягового модуля.

После подстановки в уравнения Лагранжа второго рода выражений кинетической и потенциальной энергий, функции рассеяния и обобщенных сил получена система нелинейных дифференциальных уравнений, описывающая движение четырехосного автономного тягового модуля по прямолинейным и криволинейным участкам железнодорожного пути и представляющая собой систему обыкновенных нелинейных дифференциальных уравнений 100-го порядка.

Для решения полученной системы дифференциальных уравнений автором разрабатывается специальное программное обеспечение, которое позволяет осуществлять комплексные теоретические исследования пространственных колебаний четырехосного автономного тягового модуля для определения показателей его динамической нагруженности и безопасности движения.

\section{Выводы}

1. Выбрана базовая расчетная схема автономного тягового модуля, которая построена как механическая система, состоящая из девятнадцати твердых тел: кузов, две рамы, четыре двигателя, четыре колесных пары и восемь приведенных масс пути, имеющих только вертикальные и попе- речные горизонтальные перемещения. При этом система имеет 82 степени свободы.

2. Проведено математическое описание возможных колебаний тел системы. При описании движения рассматриваемого экипажа по участкам пути произвольного очертания выбрана неподвижная система координат, а для каждого твердого тела - по две подвижные системы координат. При выводе уравнений движения использованы общепринятые обозначения перемещений отдельных тел системы.

3. Определены ограничения и виды связей между телами системы, включая взаимодействие экипажа и рельсового пути. При этом учтены общепринятые допущения и конструктивные особенности ходовых частей автономного тягового модуля.

4. Составлены системы дифференциальных уравнений пространственных колебаний автономного тягового модуля в четырехосном исполнении.

Таким образом, автором предложено описание оригинальной математической модели пространственных колебаний четырехосного автономного тягового модуля при его движении по прямолинейным и криволинейным участкам железнодорожного пути. При этом конструкция четырехосного автономного тягового модуля представлена как сложная механическая система, а путь представляет собой упруго-вязкую инерционную систему. Данная математическая модель является базовой для проведения дальнейших теоретических исследований динамики автономных тяговых модулей при одиночном движении или при движении в составе поезда.

\section{Список использованных источников}

1. Мямлин С. В., Недужая Л. А. Совершенствование конструкции ходовых частей локомотивов. Наука та прогрес транспорту. Вісн. Дніпропетр. нац. ун-ту залізн. трансп. ім. акад. В. Лазаряна. 2013. № 5 (47). C. 124-136. DOI: 10.15802/stp2013/17977.

2. Мямлин С. В., Лунис О., Недужая Л. А. Особенности конструкции ходовых частей тягового подвижного состава. Наука та прогрес транспорту. Вісн. Дніпропетр. нац. ун-ту залізн. трансп. ім. акад. В. Лазаряна. 2017. № 3 (69). C. 130-146. DOI: 10.15802/stp2017/104824. 
3. Spiryagin M., Wolfs P., Cole C., Spiryagin V., Sun Y. Q., McSweeney T. Design of Mechanical Systems of Locomotives from: Design and Simulation of Heavy Haul Locomotives and Trains. CRC Press. 2016. 477 p.

4. Радченко Н. А. Криволинейное движение рельсовых транспортных средств. Київ: Наукова думка, 1988. 212 с. $528 \mathrm{c.}$

5. Лазарян В. А. Динамика транспортных средств: избр. тр. Київ: Наукова думка, 1985.

6. Garg V., Dukkipati R. Dynamics of Railway Vehicle Systems. Toronto: Academic press, 1984. $407 \mathrm{p}$.

7. Автоколебания и устойчивость движения рельсовых экипажей / Ю. В. Демин, Л. А. Длугач, М. Л. Коротенко, О. М. Маркова. Київ: Наукова думка, 1984. 160 с.

8. Математическое моделирование колебаний рельсовых транспортных средств / В. Ф. Ушкалов, Л. М. Резников, В. С. Иккол, Е. Ю. Трубицкая, С. Ф. Редько, А. И. Залесский. Київ: Наукова думка, 1989. 240 с.

9. Kalker J. J. Three-dimensional elastic bodies in rolling contact. Springer Netherlands, 1990. 314 p. DOI 10.1007/978-94-015-7889-9.

10. Myamlin S., Luchanin M., Neduzha L. Construction Analysis of Mechanical Parts of Locomoties. TEKA. Commision of Motorization and Power Indastry in Agriculture. 2013. Vol. 13, № 3. P. 162-169.

11. Ткаченко В. П., Малюк С. В. Моделювання руху традиційної колісної пари в прямих ділянках колії та доцільність ії модернізації. Логістичне управління та безпека руху на mранспорті: матеріали науково-практ. конф. 5-7 жовтня 2017 р., м. Лиман (Донецька обл.). Сєвєродонецьк : СНУ ім. В. Даля, 2017. С. 169-172.

12. Нерубацький В. П., Плахтій О. А., Гордієнко Д. А. Розробка тривимірної моделі системи «рама візка - тягові електродвигуни» для електровоза ЧС4 за допомогою програмного продукту SolidWorks. Інформаційно-керуючі системи на залізничному транспорті. 2018. № 4. C. 21-29.

13. Буйносов А. П., Тихонов В. А. Разработка математической модели механической части электровоза ВЛ11К. Технологическое обеспечение ремонта и повышение динамических качеств железнодорожного подвижного состава: материалы всеросс. научно-техн. конф. с междунар. участием, 10-11 ноября 2011 г. Омск: Омский гос.ун-т путей сообщения, 2011. С. 33-38.

14. Коссов В. С., Сазонов И. В. Основные направления совершенствования проектирования и производства локомотивов, позволяющие снизить стоимость их жизненного цикла. Бюллетень Объединенного ученого совета ОАО «РЖД». 2016. № 5. С. 5-12.

15. Моделирование системы «электроподвижной состав-тяговая сеть» и влияние на процессы управления движением субъективных факторов / В. И. Омельяненко, Н. Н. Калюжный, В. Е. Бондаренко, Л. В. Оверьянова. Вестник Нац. техн. ун-та «ХПИ»: сб. науч. трудов. Темат. вып.: Транспортное машиностроение. Харьков: НТУ «ХПИ». 2011. № 18. C. $75-83$.

16. Орлов Е. А. Параметрический подход к моделированию динамики железнодорожных экипажей. Вестник Наи. техн. ун-та «ХПИ»: сб. науч. трудов. Темат. вып.: Машиноведение и САПР. Харьков: НТУ «ХПИ», 2006. № 33. С. 77-87.

17. Wu Q., Cole C., Luo S., Spiryagin M. A review of dynamics modelling of friction draft gear. Vehicle System Dynamics. 2014. Vol. 52, №. 6. P. 733-758. URL: https://doi.org/ 10.1080/00423114.2014.894199.

Буреш Франтишек, АО «Украинские железные дороги», член правления. ORCID ID: 0000-0003-2171-7476. Тел. +38-044-465-00-41. 
Збірник наукових праць Українського державного університету залізничного транспорту

František Bures, Joint stock company «Ukrainian Railways», Member of the Board. ORCID ID: 0000-0003-2171-7476. Tel. +38-044-465-00-41.

Статтю прийнято 26.11.2020 p. 